\title{
STUDY OF CLINICAL PRESENTATION THERAPEUTIC RESPONSE IN PHRYNODERMA CASES.
}

Kallappa H, Raina Jain, Raghu M. T, Lalitha C.

1. Professor and HOD, Department of Dermatology and Venereology, Navodaya Medical College and Research Centre

2. Junior Resident, Department of Dermatology and Venereology, Navodaya Medical College and Research Centre

3. Assistant Professor, Department of Dermatology and Venereology, Navodaya Medical College and Research Centre

4. Assistant Professor, Department of Dermatology and Venereology, Navodaya Medical College and Research Centre

\author{
CORRESPONDING AUTHOR: \\ Kallappa C, \\ Professor and HOD \\ Department of Dermatolgy, \\ Navodaya Medical College and Research Centre. \\ Raichur, Karnataka. Pin: 584103. \\ E-mail: drkallappa@gmail.com
}

\begin{abstract}
BACKGROUND AND OBJECTIVES: Phrynoderma is a form of follicular hyperkeratosis that is associated with nutritional deficiencies. It is endemic to poor population and constitutes significant cases in dermatological clinics in India. The present study is undertaken to find out the therapeutic response in Phrynoderma cases. METHODS: A total of 100 patients with Phrynoderma after clinical diagnosis, who consented for study were included. Patients were divided into three groups of 34, 33 and 33 each. Following treatment regimens were given for each group.

R1: Oral Vitamin A 50,000 IU daily for 8 weeks

R2: Oral Vitamin E 100 IU daily for 8 weeks

R3: Safflower Oil 1 tsp TD w/ meal for 1 month

RESULTS: In a total of 100 patients of Phrynoderma studied; incidence was found to be $0.45 \%$ of which males were $63 \%$ and females were $37 \%$. The male to female ratio was 1.70:1. Therapeutic response was better in R1 (Oral Vitamin A) group compared to R2 (Oral Vitamin E) group. Amongst R2 and R3 (safflower Oil), R2 showed better response compared to R3.
\end{abstract}

KEYWORDS: phrynoderma, follicular hyperkeratosis, Safflower oil, therapeutic interventions

INTRODUCTION: Phrynoderma is a form of follicular hyperkeratosis that is associated with nutritional deficiencies. Phrynoderma occurs usually in children and adolescents and is characterized by hyperkeratotic, follicular papules with keratotic plug distributed over elbows, knees, extensor extremities and buttocks, though any part of the body may be involved. ${ }^{1}$ The term Phrynoderma, meaning "toad skin", was first coined by Lucius Nicholls in 1933, who observed follicular hyperkeratosis in African laborers with vitamin A deficiency. ${ }^{2}$

Deficiencies of Vitamin A, Vitamin B, Vitamin C, Vitamin E and essential fatty acid are considered as etiological factors for this form of dermatosis.1, 3 Phrynoderma is believed to be a manifestation of severe malnutrition, and the clinical picture typically improves with enhanced 
nutritional status. Phrynoderma is unquestionably a disorder of nutritional deficiency and it has been included as a sign to be looked for in the schedule for the assessment of nutritional status of individuals and population. ${ }^{4}$

It is rare in developed countries but common amongst poor populations. Phrynoderma may occur as a result of malabsorption due to surgical or medical causes, such as small-bowel bypass surgery, colectomy and pancreatic insufficiency, which are more commonly seen in developed countries. ${ }^{5}$ The present study is undertaken to evaluate the therapeutic response of phrynoderma to different nutritional agents.

METHODS: The source of data is from patients attending skin outpatient department and other patients admitted in Navodaya Medical College Hospital and Research Centre, Raichur. One hundred consenting consecutive cases have been taken for study. An informed consent was taken from all patients. The period extended from October 2010 to September 2012.

A detailed history was taken, regarding the age, sex, socioeconomic status, family history, recurrences and duration of symptoms. The socio-economic status was classified based on income group developed by National Council of Applied Economic Research, New Delhi in 1993-94. Accordingly, patients were divided into low/ middle/ high income groups. A detailed examination of the cutaneous lesions was done in every patient including description of morphology, shape, size, color, keratotic plug and issuing hair), site of involvement, distribution-pattern, site of onset and changes in adjacent skin was carried out. Diagnosis was made on clinical features. The disease was considered localized if lesions were restricted to elbows, knees, buttocks and extensor extremities, and generalized when back/ face/ neck were involved. Wherever required, ophthalmic and paediatric opinion was taken. All patients underwent following lab tests: Hemoglobin ( $\mathrm{Hb} \%)$, total and differential white blood cell counts (TLC, DLC) and routine urine examination (urine protein, sugar, and microscopy). No other lab tests were done unless deemed necessary. Since majority of patients in the present study were young children, most of their parents refused for biopsy. Hence, histopathological studies were carried out for 20 patients with their prior consent.

In methods, as essential fatty acids are generally received in adequate amount from cooking oil, the Patients were tested with Vitamin A, Vitamin E and Safflower Oil. Also, all the patients received topical keratolytics- salicylic acid 3\% and tretinoin $0.025 \%$ daily. Following regimens were given for a group of $1 / 3^{\text {rd }}$ patients each ( 41 patients each).

R1: Oral Vitamin A 50,000 IU daily for 8 weeks

R2: Oral Vitamin E 100 IU daily for 8 weeks

R3: Safflower Oil 1 tsp TD w/ meal for 1 month

During the treatment period, patients were asked to follow the same diet without any modification. Initial severity of involvement was assessed in the form of total area of involvement in square centimeters. Total area of involvement was re-assessed at the end of two months. The percentage of improvement (reduction in the total involved area after two months) was graded against their response as below: 
Nil $\quad: 0 \%$ Improvement

Poor $\quad:<25 \%$ Improvement

Moderate : :26 49\% Improvement

Good $\quad:$ 50 74\% Improvement

Excellent $\quad:>75 \%$ Improvement

The collected data was interpreted and analyzed statistically for therapeutic response. In the statistical analysis, frequency variables were presented as numbers and percentages. They were analyzed by chi-square test and Fisher's exact test. A p-value of 0.05 or less was considered for statistical significance.

RESULT: Among the 100 patients, $63 \%$ were male and 37\% were female (M:F=1.70:1). Majority of patients were in the age group of 5 10 (59\%), followed by age group of 11 15 (29\%), then age group of 16 20 (5\%) and for the age group greater than 20 years (3\%). Majority of patients (89\%) were from the lower income stratum and rest from middle income stratum. There were no patients from higher income group. 92\% of these were students, with remaining include pregnant women, labourers and housewives etc. Only 3\% patients gave history among siblings in their family. Past history was present in $9 \%$ patients

The ratio of patients compared for seasonal variation showed Winter: Monsoon: Summer = 48:44:8. Majority of cases (84\%) were presented within 6 months of onset of disease.

$92 \%$ patients presented were asymptomatic and remaining $8 \%$ complained of itching. The disease was localized in $85 \%$ cases and generalized in 15\% cases. The ratio of patients presenting bilaterally symmetrical pattern to bilaterally asymmetrical pattern was 71:29. Similarly, the ratio of grouped lesions to scattered lesions was 80:20.

The lesions were follicular, discrete, pihead sized and acuminate in all the patients. Both pigmented and skin colored lesions; and keratotic plugs with broken hair were seen in all the patients. When touched, they all felt like nutmeg grater.

Elbow was the site of onset (first site for the occurrence of lesions) for 85\%, followed by knee $7 \%$, Buttock 4\%, Thighs $2 \%$, Forearm and Legs 1\% each. The surrounding skin was normal in $6 \%$ cases, pigmented in $55 \%$ and Dry or Scaly in 31\% patients.

$85 \%$ of the patients were anemic, 10\% had Pityriasis Simplex, 9\% had Angular Stomatitis/ Glosstis. $4 \%$ had signs of Vitamin-A deficiency. Other conditions associated were Xerosis (2\%), Ichthyosis (1\%), Helminthiasis (2\%), Upperer Respiratory Tract Infections (4\%) and Gastro Enteritis (4\%).

Among the 20 biopsy specimen studied, the epidermal changes were noted in all cases and they were Hyperkeratosis $100 \%$, Acanthosis $80 \%$ and Parakeratosis 15\%. Follicular Hyperkeratosis and Plugging with compact keratin were seen in all cases i.e. 100\%. Sebaceous Gland Atrophy were present in $20 \%$ cases. Perifollicular and perivascular infiltration were $80 \%$ and $100 \%$ respectively.

In Regimen 1, around 88\% patients showed good to excellent response to the treatment, followed by $9 \%$ showing moderate response and 3\% showed poor response.

In Regimen 2, 67\% showed poor response to the treatment, followed by $18 \%$ showing moderate response and the rest $15 \%$ showing good to excellent response.

In Regimen 3, 61\% showed moderate response to the treatment, followed by $27 \%$ showing good to excellent response and remaining $12 \%$ showed poor response. 


\section{ORIGINAL ARTICLE}

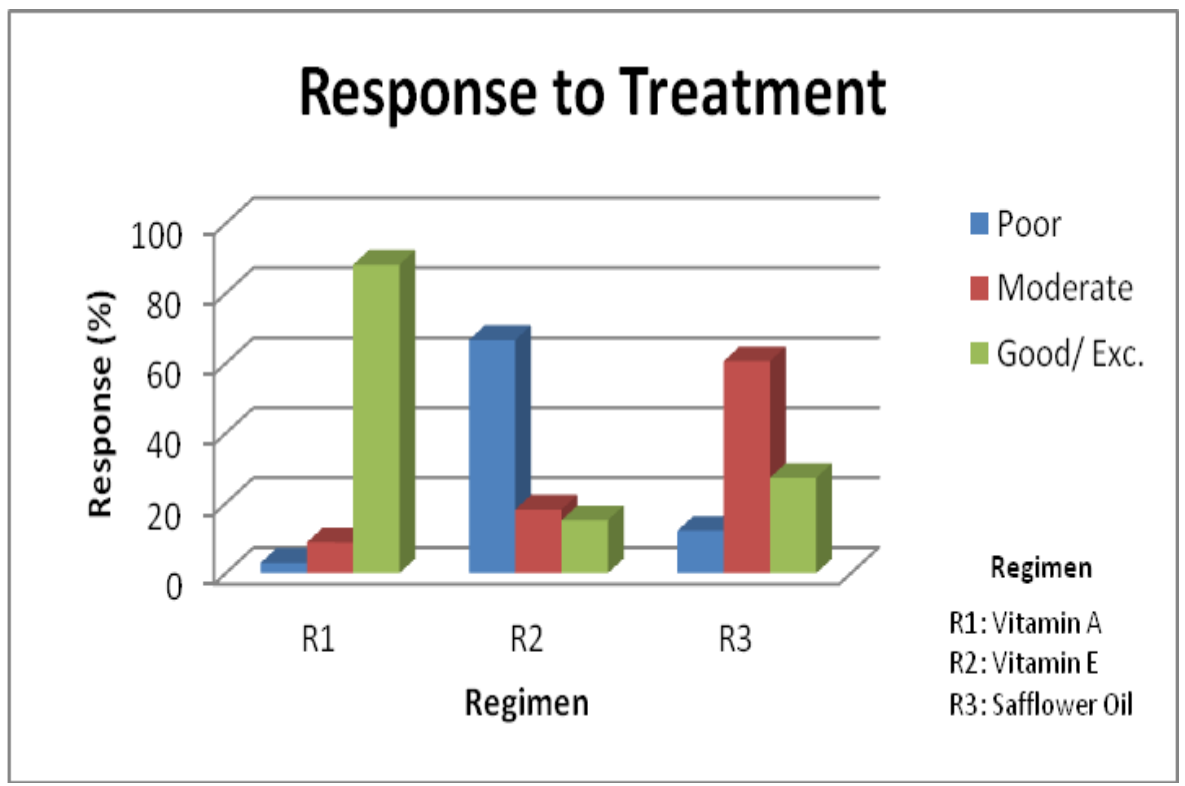

Table-1.Response to Treatment

Statistically significant difference in response was noted among the groups $(\mathrm{p}<0.01)$. On comparing the response of each regimen with other, it was noted that regimen 1 showed significantly better response compared to regimen 2 and 3, whereas regimen 3 showed significantly better response when compared to regimen 2 .

\begin{tabular}{|c|c|c|}
\hline Regimen Compared & $\mathbf{X}^{\mathbf{2}}$ & $\mathbf{P}$ \\
\hline R1 Vs R2 & 38.02 & $<0.01$ \\
\hline R1 Vs R3 & 25.66 & $<0.01$ \\
\hline R2 Vs R3 & 21.14 & $<0.01$ \\
\hline
\end{tabular}

Comparison of 3 Regimens

DISCUSSION: Hyperkeratotic follicular papules are seen in many other dermatological disorders and mild degree of follicular prominence may be seen during winter season, especially in children. The etiology of disease is still controversial and the response to various therapies is varied. Therefore in the present study of 100 patients of Phrynoderma, clinical presentation/ therapeutic response/ histopathological features were noted. In the following section, an effort is made to discuss the saliant clinical and histopathological features of Phrynoderma and it's response to regimens used in the study. This may help to differentiate it from other follicular keratoses and elucidate causative nutritional deficiency.

INCIDENCE: Total no of patients screened was 22223 ( OPD patients and in-patients during the study period) out of which, 100 patients were diagnosed with Phrynoderma. So, in the present 
study, the incidence of Phrynoderma patients cases was $0.45 \%$, which is less compared to other studies, where it was found to be $1.3 \%, 3 \%$ and $5 \% .6-8$ This is because the incidence of Phrynoderma varies depending on the ethnic groups, geographic and environmental conditions. ${ }^{2}$ Moreover, these studies were conducted in the middle part of last century. In a recent study ${ }^{17}$, incidence was found to be $0.51 \%$. Relatively less proportion of Phrynoderma cases in the present study may be due to improved nutritional status and implementation of various nutritional programmes.

SEX: In the present study, males (63\%) were affected, more than the females (37\%). Some studies have shown male preponderence.6,14 while others have shown female preponderence.8,9 Therefore, it does not seem likely that there is any inherent difference between the sexes in development of Phrynoderma.

AGE: Phrynoderma is uncommon below 5 years of age as well as above 15 years of age. In this study, majority i.e. $88 \%$ of the patients were in the age group of $5 \sim 15$ years, which is similar to other studies. 6,14

SOCIO-ECONOMIC STATUS: In the present study, majority of Phrynoderma patients were from lower income group (89\%) and the rest were from middle income group (11\%). This is similar to other studies where majority of patients were from lower socio-economic status. ${ }^{6}$

OCCUPATION: In the present study, students (92\%) were more commonly affected since majority of patients were in the age group of 5 15 years. Apart from students, housewives and pregnant women constituted $2 \%$ and labourers $2 \%$., while no significance could be drawn with reference to occuption.

FAMILY HISTORY: In the present study, family history of Phrynoderma especially in siblings was $3 \%$ of patients which is comparable with other studies . ${ }^{6-8}$ Absence of disease in siblings who were also taking same diet may suggest that apart from dietary factors, other factors play a role in development of Phrynoderma. 8 It is also posiible that dietary habits be different in siblings, even though they are provided with the same diet by parents, for eg. one sibling like a particular food, so he is taking it more and other sibling taking it less due to dislike.

SEASONAL VARIATION: In the present study, only $8 \%$ of the patients were presented during summer. Almost equal percentage of patients was presented during monsoon and winter season viz. $44 \%$ and $48 \%$ respectively. This is in accordance with the fact that the incidence of phrynoderma is higher in colder months of year.

DURATION: In the present study, majority of the patients (84\%) presented within 6 months of appearance of lesions, which is comparable to other study (90.1\%). ${ }^{6}$ In the present study, duration of the disease more than 1 year was relatively rare $5 \%$, which is again comparable with the other study $(6.17 \%) .{ }^{6}$ Patients usually present between 2 weeks to 1 year, after the onset of disease. ${ }^{6-8}$ 
SYMPTOMS: In the present study, majority (92\%) of the patients were asymptomatic. The remaining patients $(8 \%)$ complained of itching. Although slight itching was reported in other studies subjective symptoms are usually absent even after asking leading questions regarding itching, pain and tenderness. ${ }^{6}$

DISTRIBUTION AND PATTERN: In the present study, majority of the lesions were localized (85\%), symmetrically distributed (71\%) and arranged in groups (80\%), especially around bony prominences. This is in accordance with the observation made by many authors, according to whom the distribution of lesions is almost always symmetrical and either localized or generalized and papules have a tendency to be arranged in groups. ${ }^{10-12,13-14}$ In the present study, some of the patients (16\%) had lesions over back. This explains the generalized (15\%) asymmetrical (29\%) and scattered (20\%) lesions noted in present study.

SITE OF ONSET: The most common site of onset was elbows (85\%), followed by knee (7\%), buttocks (4\%), rarely thighs (2\%), forearms and legs (1\%) each. This is in accordance with other studies where sites of predilection for the onset of lesions were knees, elbows, buttocks, forearms, legs, arms and thighs, in the order mentioned. Thus, Phrynoderma usually starts at the site of pressure and friction, which may act as predisposing factors. ${ }^{6}$

SITE OF INVOLVEMENT: In the present study, elbows (98\%) and knees (82\%) were affected in majority of the cases. Extensor extremities (64\%), buttocks (36\%) were the next commonest sites involved. This is in accordance with other studies, where characteristic sites of involvement in majority of cases were elbows, knees, extensor extremities and buttocks. As a part of generalized disease, back (16\%), face (6\%) and neck (5\%) were affected.6-8

MORPHOLOGY: In the present study, in all cases follicular discrete acuminate both pigmented and skin colored papules with keratotic plugs and broken hair were seen and felt like nutmeg grater. This is similar to observations in other study.

ADJACENT SKIN: In the present study, the surrounding skin (94\% of cases) was dry, scaly or pigmented, which is in accordance with other studies where similar features were noted in association with typical morphological features. .-8 $^{-8}$

ASSOCIATED DISEASE AND CONDITIONS: Anemia was present in majority of patients (85\%), which indicates general poor health and nutrition.

Association of pityriasis alba and phrynoderma may be due to the prevalence of both diseases in the same age group i.e. between 3 16 years.

In present study, signs of Vitamin B-Complex deficiency like angular stomatitis and glossitis were seen in some of the patients (9\%). As the other studies were conducted in the middle part of the last century, the higher percentage of Vitamin B-Complex deficiency signs were observed 6 . The decrease in the prevalence of Vitamin B-Complex deficiency may be due to nutritional programmes, supplementation of food and improved standard of living. 
In the present study, signs of Vitamin A deficiency were seen in $4 \%$ of the patients, which is comparable with other study showing $5 \%{ }^{7}$

In the present study, association of Phrynoderma and lactation was due to increased nutritional demand and food restrictions followed in this part of the country during pregnancy and lactation

HISTOPATHOLOGICAL CHANGES: In the present study, follicular hyperkeratosis and follicular plugging with compact keratin and mild epidermal hyperkeratosis along with perivascular infiltration were seen in all the cases. Mild acanthosis and perifollicular infiltration were seen in $80 \%$ patients.

Lymphocytic infiltrate was moderate in $60 \%$ of the patients and few in $40 \%$ of the patients. Sebaceous gland atrophy was seen in $20 \%$ patients; the reason may be that sebaceous gland atrophy is seen only in the later stage in typical papules of Phrynoderma and moreover serial sections are required to demonstrate the same. The parakeratosis present in $15 \%$ of the cases is comparable with other studies. ${ }^{6}$

RESPONSE TO TREATMENT: In the present study, the response to Vitamin-A therapy was good to excellent in $88 \%$ of patients. This is comparable with other studies where treatment with Vitamin A $50,000 \sim 150,000$ IUs daily cleared the lesions in 1 4 months. ${ }^{9-11}$ Similar good response has been reported with cod-liver oil, also a good source of EFA.

Good response to Vitamin-A seen in the present study may be due to anti-keratinizing effect of Vitamin A in the doses of 50,000 IUs daily or higher. The role of Vitamin A deficiency in Phrynoderma couldn't be proved conclusively in the present study, because of lack of biochemical evidences. However, Vitamin A is definitely effective in the treatment of the lesions. ${ }^{15-16}$

In the present study, the response to Vitamin E therapy was poor in $66.67 \%$ cases and only $15 \%$ patients showed good to excellent response, which is lower than the response noticed in other studies. ${ }^{16}$ In the present study, the role of Vitamin E couldn't be excluded conclusively.

In the present study, the response to Safflower Oil was moderate in $60.61 \%$ patients and good to excellent in $27.27 \%$ of patients. Safflower oil is rich in oleic acid and linoleic acid.

Table 2.

Typical Fatty Acid Composition of Safflower Oil

Fatty Acid Percentage Composition

Palmitic $\quad 5.25$

Stearic $\quad 1.50$

Oleic $\quad 15.00$

Linoleic $\quad 77.00$

Others $\quad 1.25$

The reason for good response to Safflower Oil in other studies probably is: different dosage and duration of the treatment. Moreover, Safflower Oil is also rich in Vitamin B-Complex and the response has been only moderate in majority of our cases. The reason may be that the response to Oral Vitamin B-Complex is poor compared to parenteral root. ${ }^{6}$ Since, the poorest Indian diet 
provides sufficient EFA, and Vitamin B-Complex are required for EFA metabolism, the EFAs may not play a direct role in the etiology of Phrynoderma, but may secondarily be affected due to Vitamin BComplex deficiency. The role of EFAs and Vitamin B- Complex couldn't be excluded conclusively. In the present study, along with the above regimen, topical tretinoin $0.025 \%$ and salicylic acid $3 \%$ cream were used during the treatment. The patients were asked to follow the same routine diet. Therefore, the responses obtained in the present study were mainly due to therapeutic agents.

CONCLUSION: Phrynoderma is a disease of young children and adolescents, commonly seen in patients belonging to lower income strata, clinically characterized by discrete, follicular, pinhead sized, acuminate, pigmented and skin colored papules with keratotic plugs and broken hairs. It is a chronic disease usually localized to elbows, knees, extensor extremities and buttocks.

Histopathologically, disorder is characterized by follicular hyperkeratosis and follicular plugging with compact keratotic material. Surrounding epidermis shows mild hyperkeratosis and acanthosis. Moderate lymphocytic infiltrate is usually seen around hair follicles. Sebaceous gland atrophy may be seen in few cases.

Good to excellent response to Vitamin A therapy suggest that Phrynoderma is a nutritional deficiency state.. This study proves that the therapeutic usage of Vitamin A is useful for the treatment of phrynoderma and is better when compared to the two other regimens. It may be either because Vitamin A deficiency has an etiological role in the causation of phrynoderma or because of the anti-keratinizing action of Vitamin A. A daily dose of 50,000 units or more acts as anti-keratinizing.

This study shows that the simultaneous use of Vitamin A,in the oral dose of 50,000 IU, given daily for 8 weeks, along with daily topical salicylic acid $3 \%$ and tretinoin $0.025 \%$, gives good results in the treatment of phrynoderma, and compared with this, the results given by oral intake of safflower oil or Vitamin E along with topical salicylic acid 3\% and tretinoin $0.025 \%$ is inferior.

Further studies are required to know the exact pathogenesis and the role played by different nutrients in the development of Phrynoderma, so that exact etiological factors can be elucidated.

\section{REFERENCES:}

1. Nakjang Y, Yuttanavivat T. Phrynoderma: a review of 105 cases. J Dermatol 1988; 15:531-4.

2. Nicholls L. Phrynoderma: A condition due to vitamin deficiency. Indian Medical Gazette 1933; 68:681-7.

3. Bleasel NR, Stapleton KM, Lee MS, et al. Vitamin A deficiency phrynoderma: due to malabsorption and inadequate diet. J Am Acad Dermatol 1999; 41:322-4.

4. Marron M, Allen DM, Esterly NB. Phrynoderma: A manifestation of Vitamin A deficiency? The rest of the history. Pediatr Dermatol 2005;22:60-3

5. Barr DJ, Riley RJ, Greco DJ. Bypass phrynoderma. Vitamin A deficiency associated with bowel-bypass surgery. Arch Dermatol 1984; 120:919-21.

6. Ayyangar Rama. Phrynoderma and nutritional deficiency. Ind J Dermatol Venereol Leprol 1967; 33:13-24.

7. Panja SK, Chatterjee SK, Mukherjee KL. Follicular keratosis in children. Ind J Dermatol 1970; 15:75-78. 
8. Shrank AB. Phrynoderma. Br Med J 1966, 1:29-30.(8)

9. Gopalan C. The etiology of phrynoderma. Ind Med Gaz 1947; 82:16-20.

10. Koley SK, Sen MK, Panja RK. Phrynoderma - A review. Ind J Dermatol Venereol Leprol 1975; 41(5):171-175.

11. Wechsler HL. Vitamin A deficiency following small bowel surgery for obesity. Arch Dermatol 1979; 115:73-75.(10)

12. Neil SM, Pembroke AC, du-Viver AW, Salisbury JR. Phrynoderma and perforating folliculitis due to vitamin A deficiency in diabetes. J R Soc Med 1988; 81:171-172

13. Clarke GH, Okoro AN. Follicular Hyperkeratosis. J Trop Med. Hyg. 1962;65:27.(12)

14. Srikantia SG, Pargaonkar VU. Follicular hyperkeratosis. J Trop Med Hyg. 1964; 67:295-296.

15. Rajagopal K, Chowdhury SR. Phrynoderma and some associated changes in blood lipids. Indian Med Gaz. 1952; 87:350-3.

16. Menon PS, Tulpule PG, Patwardhan VN. Phrynoderma: Clinical and Biochemical Investigations. Indian J Med Res. 1950; 38(2):173-186.

17. Ragunath S, Kumar VJ, Murughesh SB. A clinical study of 125 patients with phrynoderma, Indian J dermatol 2011; 56; 389-392.

18. Girard C, Dereure O, Blatiere V, Guillot B, Bessis D. Vitamin A deficiency phrynoderma associated with chronic giardiasis. Pediatr Dermatol 2006;23:346-9

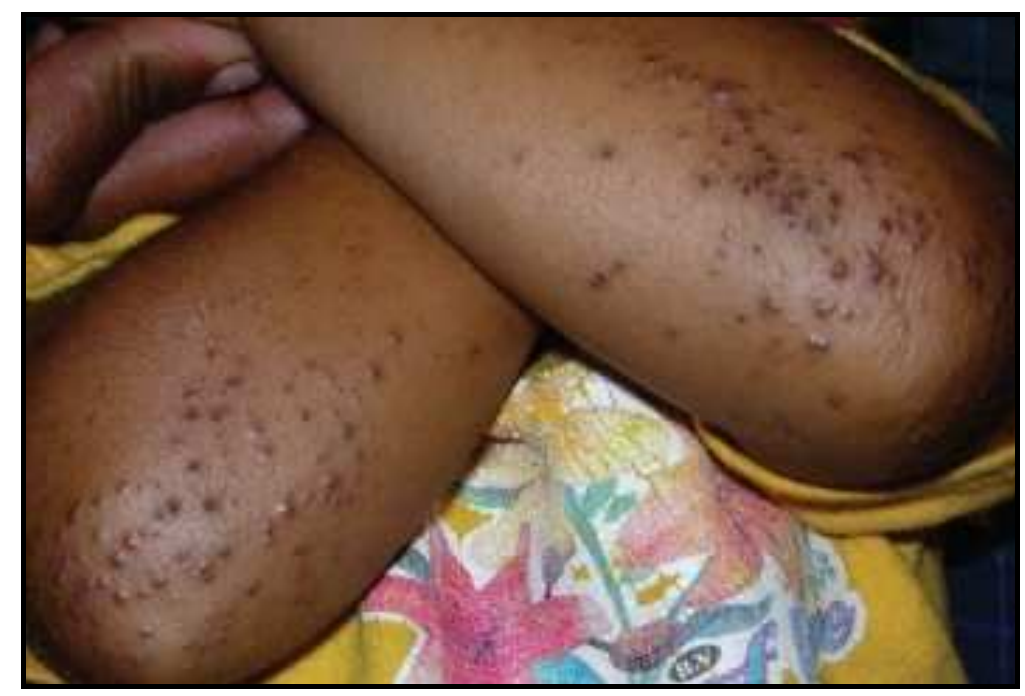

Clinical Photograph-1Before Treatment with Regimen1 with Vitamin A Therapy 


\section{ORIGINAL ARTICLE}

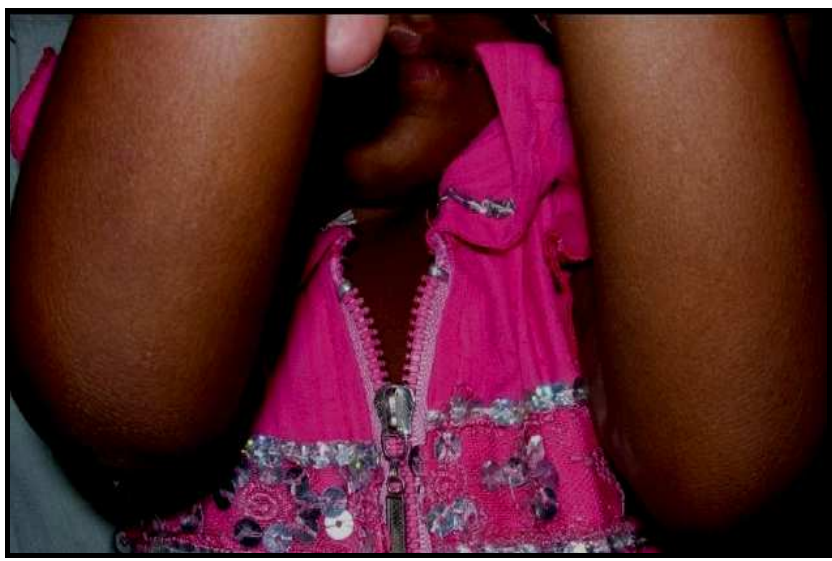

Clinical Photograph-2 After Treatment with Regimen1 with Vitamin A Therapy

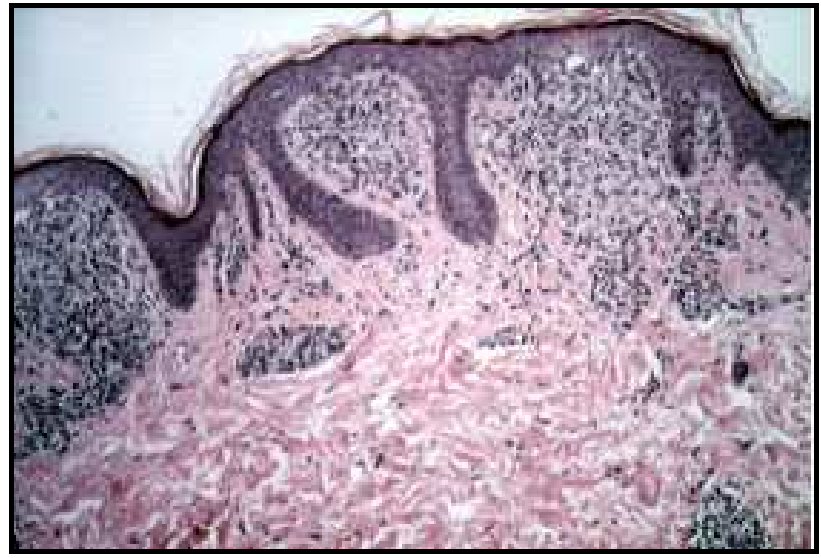

Histopathology Photomicrograph-3 Follicular Plugging with Compact Keratin and Hyperkeratosis

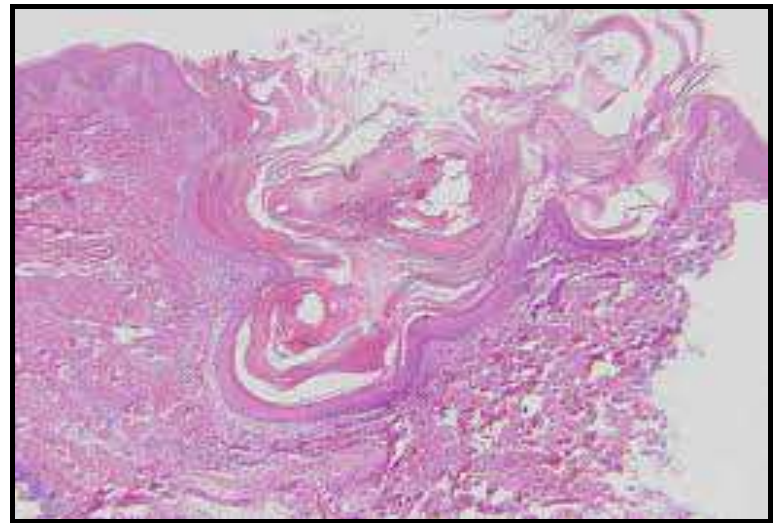

Histopathology Photomicrograph-4: Follicular Plugging with Compact Keratin and PFI. 